\title{
EGU2020-21351
}

https://doi.org/10.5194/egusphere-egu2020-21351

EGU General Assembly 2020

(c) Author(s) 2021. This work is distributed under

the Creative Commons Attribution 4.0 License.

\section{Fully-coupled 3D modelling of magmatic dike propagation - finite pulse release from a point source}

\author{
Andreas Möri, Brice Lecampion, and Haseeb Zia \\ EPFL - École polytechnique fédérale de Lausanne, Lausanne, Switzerland (andreas.mori@epfl.ch)
}

Magmatic dikes are a naturally occurring type of fluid-driven fractures [1] propagating in the lithosphere driven by buoyancy (more precisely by the difference between the in-situ minimum horizontal stress gradient and the magma weight). Fully-coupled modelling of these 3D fractures is very challenging and most contributions until today have been restricted to $2 \mathrm{D}$ plane-strain. These 2D investigations have highlighted the importance of the head-tail structure, notably the fact that lubrication flow in the tail is driving the growth of the hydrostatic head $[2,3]$. We investigate the 3D development of a buoyant dike from a point source, focusing on the case of a finite volume release under homogeneous conditions (homogeneous material properties and buoyancy contrast). We use the fully coupled planar 3D hydraulic fracture growth solver PyFrac based on the implicit level set algorithm [4].

This configuration shows an early time behaviour heavily dominated by the effects of the pulse release. The initially radial hydraulic fracture transitions toward a large time buoyant dike solution. At large time our simulations tends to the finger-like/constant breadth solution [5] albeit extremely slowly. Our results confirm the 3D toughness dominated head structure and the importance of the viscous tail as the driving mechanism for the dynamics of such a 3D Weertman's pulse (form of the head). Depending on the initial phase of the pulse release, we observe an overshoot of the dike breadth when it is initially strongly dominated by viscous dissipation. Using a scaling analysis, we characterize the transition from the early time radial finite pulse fracture to the late dike constant breadth solution. Our simulations show, that the time when the buoyant force takes its full dominance is crucial and governs the existence (or not) of an overshoot. Mainly we show that the overshoot depends on a transitional time/lengthscale. A detailed understanding of the fracture propagation after the end of the finite volume release (yet without buoyancy) is key to quantify this lengthscale. We thus present scalings and semi-analytical solutions for this case and discuss its relevance for the transition toward a buoyancy driven dike propagation.

[1] E. Rivalta, B. Taisne, A.P. Bunger, and R.F. Katz. Tectonophysics, 638:1-42, 2015.

[2] J. R. Lister and R. C. Kerr. J. Geohpys. Res. Solid Earth, 96(B6):10049-10077, 1991.

[3] S. M. Roper and J. R. Lister. J. Fluid Mech., 536:79-98, 2005.

[4] A. P. Peirce and E. Detournay. Comput. Methods in Appl. Mech. Eng., 197(33-40):2858-2885, 
2008.

[5] L.N. Germanovich, D. I. Garagash, Murdoch, L., and Robinowitz M. AGU Fall meeting, 2014. 